Dani J. Barrington*

\title{
Inadequate water, sanitation and hygiene in the South Pacific: how might it be impacting children?
}

DOI 10.1515/reveh-2015-0034

Received October 7, 2015; accepted October 13, 2015; previously published online January 20, 2016

\begin{abstract}
It is detrimental to anyone's health to live with conditions of inadequate water, sanitation and hygiene (WaSH). Research suggests that the impact is greatest on children, and that poor WaSH conditions during the formative years can lead to decreased physical, mental and social well-being throughout one's life. Little research has investigated how such poor WaSH conditions are negatively impacting children in the South Pacific region, and hence contributing to disease and social burden. To increase children's opportunities to develop physically and intellectually in a healthy and sustained manner, it is important that practitioners take a holistic approach to improving WaSH by acknowledging it as a core component of environmental health.
\end{abstract}

Keywords: enteropathy; Fiji; stunting; WaSH.

\section{Background}

Inadequate water, sanitation and hygiene (WaSH) are known to be significant contributors to physical disease in low and middle income countries (1), and WaSH interventions are hence common throughout the developing world. There is an ongoing discourse throughout the WaSH sector as to the differences between 'sanitation' and 'hygiene', with the majority of WaSH programs interpreting the two as some mixture of excreta management as well as solid waste management, personal hygiene as indicated by handwashing, bathing and menstrual hygiene management.

From an environmental and public health perspective it is not important how different facets of water, sanitation and hygiene are categorized. What is significant is that all

*Corresponding author: Dani J. Barrington, Department of Marketing, Monash University, Wellington Road, Clayton 3800, VIC, Australia; International WaterCentre, Level 16,

333 Ann Street, Brisbane 4000, QLD, Australia; and School of Public Health, The University of Queensland, Herston Road, Herston 4006, QLD, Australia, Phone: +(61) 412528 402,

E-mail: dani.barrington@monash.edu three aspects are important contributors to the healthy environments we need to live a healthy life through all stages of our development. Research outcomes have shown that the impact of poor $\mathrm{WaSH}$ on children in particular can have a lasting detrimental impact throughout their adult years and place an ever growing burden of disease on individual, community and country well-being (2).

In the South Pacific, very little peer-reviewed research has been published on the detrimental impacts of poor WaSH in general, let alone on children (3). Yet many children in the South Pacific live in dire WaSH conditions which may significantly impact upon their physical and intellectual development (Figure 1). WaSH interventions by governmental agencies and community service organizations (CSOs) are common, but there is a lack of information available in the public domain surrounding whether these programs have improved health outcomes.

\section{WaSH and physical development}

Poor WaSH conditions contribute to many diseases which can have long term impacts on children, particularly where they lead to permanent disabilities. Common diseases found in unhygienic WaSH conditions include those which are caused by vectors that breed in stagnant water and waterways (e.g. malaria, dengue, schistosomiasis), the ingestion of pathogens (e.g. cholera, ascariasis), and the penetration of the skin (e.g. hookworm infections) (1, 4). Many of these diseases are known to cause diarrhea, and the most common health measurement for assessing the potential impact of WaSH interventions is by measuring diarrheal episodes and investigating whether the WaSH project has led to a decrease in frequency.

It is common for children in poor WaSH environments to be exposed on a daily basis to human fecal matter (e.g. people not using a toilet, the toilet is not integrated into an appropriate waste treatment system, e.g. Figure 1, there is a lack of handwashing, watery diarrhea is not well contained) (5), as well as that of various animals which often roam free around the household (e.g. dogs, chickens) (6). Prolonged exposure to fecal 


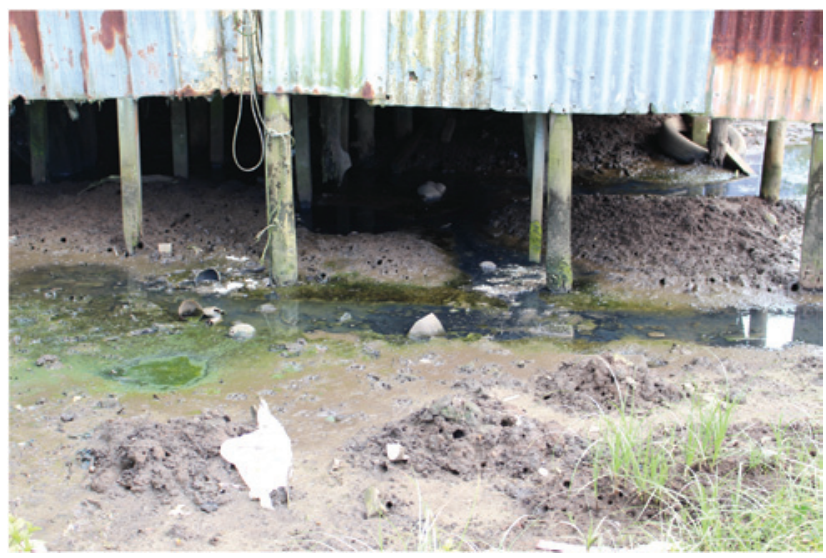

Figure 1: Household wastewater pools around a home in the South Pacific. This household has a pour-flush toilet, but the collection tank does not contain the wastewater during daily tidal flooding. Children play in such areas around households (Photograph by Dani J. Barrington).

material results in environmental enteropathy, which causes morphological changes to the gastrointestinal tract, and is known to induce malnutrition and diarrhea (7). Alongside these impacts, environmental enteropathy is thought to contribute to stunting in the first 1000 days following conception (8). Stunting results not just in lower than average height-for-age ratios and physical health issues, but also reduced cognitive function throughout the lives of those affected $(9,10)$. Thus environmental enteropathy is linked to poor WaSH conditions $(6,11)$ and estimating the detrimental impacts of poor WaSH by diarrheal diseases alone may underestimate the burden inadequate $\mathrm{WaSH}$ is placing on society (7).

There are many regions within the South Pacific where open defecation is commonplace, alongside other poor WaSH conditions, and where many diseases known to be related to inadequate WaSH conditions are common [e.g. cholera (12)]. Although there has been some research into the short term impacts of these diseases, no research has yet investigated how these WaSH related diseases impact the long term physical development of children in the South Pacific, and what burden this in turn places on society.

\section{WaSH and intellectual development}

A reduction in cognitive function caused by stunting can significantly impact not just physical well-being, but also mental and social well-being, which are considered key determinant's of one's health by the World Health Organization (13). Detrimental impacts on cognitive function can lead to reduced educational performance, and in turn less employment opportunities and a greater probability of living in poverty, impacting these children throughout adulthood (9), and often perpetuating the cycle of poor WaSH conditions and stunting of their own children. Reduced educational performance can be compounded by poor WaSH conditions present in the school environment.

Most children over the age of 5 spend a large proportion of their time in school (93\% of children of primary school age are enrolled in primary education) (14). The WaSH conditions of schools are thus very important not just for preventing physical diseases, but for ensuring that children gain an education. Yet $29 \%$ of schools globally still do not provide accessible drinking water, $31 \%$ do not provide adequate sanitation facilities, and $79 \%$ do not provide adequate handwashing facilities to students (15).

Similarly to the home environment, poor WaSH conditions in schools can result in physical illnesses, caused not only by pathogens present in unsanitary environments, but also the practice of 'holding oneself in' so that students do not urinate or defecate during school hours and instead wait until they can return home to use the toilet (16). Conversely, where WaSH facilities are available at schools but not in the household, anecdotal evidence has shown a disruption to learning from children frequently leaving the classroom to relieve themselves during lessons, rather than spending their recess and lunch periods waiting in lines to use the facilities.

School absenteeism leads to reduced time spent in education, which can have significant impacts on social and mental well-being throughout one's life. Absenteeism due to physical illnesses caused by poor WaSH conditions within the household or school is common, but absenteeism also occurs for non-physical reasons (16). The most prominent examples in the literature refer to the large proportion of girls leaving school when they reach puberty, frequently citing lack of toilets or handwashing facilities as reasons. Even those girls who do not drop out of school entirely are known to miss several days per month when menstruating, not just because of a lack of available hygienic menstrual management, but also because many WaSH facilities are not private and may result in teasing from boys or other girls (17). Clearly access to adequate WaSH within both the household and school environments is key to ensuring that children are able to participate in educational activities so as to develop intellectually. 


\section{An environmental health approach to WaSH in the South Pacific}

Reflecting on the Millennium Development Goals in order to prepare their successor, the Sustainable Development Goals, WaSH experts across the globe have agreed that alongside providing access to water and toilets, there is a need to improve hygiene, particularly for women and girls (18). The sector has observed over many years that water that is safe at the source is often polluted by household practices of carriage and storage (19), providing free toilets will not result in the cessation of open defecation if it is the preferred sanitation option of households (20), and handwashing is not a simple behavior to instil (19). Furthermore, even if one does drink safe water, use a toilet, and wash their hands at the five critical times, one is not necessarily safe from the dangers posed by poor WaSH conditions, particularly if households around theirs continue to practice inadequate WaSH behaviors (21). The author can attest to this personally as she drinks only treated water, uses improved sanitation and manages her personal hygiene, yet continues to be plagued by gastro-intestinal illness on most fieldtrips to visit communities in the South Pacific.

The WaSH sector has traditionally been dominated by engineers, and is not short of appropriate water and sanitation technologies (22). Many projects in the South Pacific have focussed on technical improvements to water supply, household toilets, and wastewater carriage and treatment, but less have taken a holistic view of working towards a health-promoting environment. Although infrastructural improvements are of course important for improving health (23), it is critical that when designing WaSH interventions practitioners do not simply focus on water, sanitation or hygiene, or even combinations of discrete activities representing each letter of the acronym. Although there can be health improvements using these methods, until WaSH is considered a holistic component of environmental health, it is unlikely that we will observe the full potential of children living in developing communities of the South Pacific. This approach of linking WaSH with environmental health (commonly referred to as 'total sanitation') is beginning to be reflected in national level policy documents [e.g. Papua New Guinea (24)]. It will be interesting to see how these are implemented in reality and whether they achieve improvements in child health.

\section{References}

1. Prüss-Ustün A, Bartram J, Clasen T, Colford JM, Cumming O, et al. Burden of disease from inadequate water, sanitation and hygiene in low- and middle-income settings: A retrospective analysis of data from 145 countries. Trop Med Int Health 2014;19(8):894-905.

2. Mara D, Lane J, Scott B, Trouba D. Sanitation and health. PLoS Med 2010;7(11):e1000363.

3. MacDonald MC, Chan T, Hadwen WL, Souter R, Kearton A, et al. Systematic review of water, sanitation and hygiene research in Small Island Developing States of the Pacific Region, in prep.

4. Bartlett S. Water, sanitation and urban children: The need to go beyond "improved" provision. Environ Urban 2003;15(2): 57-70.

5. Fagundes-Neto U, Viaro T, Wehba J, Patricio FRdS, Machado NL. Tropical enteropathy (environmental enteropathy) in early childhood: A syndrome caused by contaminated environment. J Trop Pediatrics 1984;30(4):204-9.

6. Schmidt CW. Beyond malnutrition: The role of sanitation in stunted growth. Environ Health Persp 2014;122(11):A298-303.

7. Humphrey JH. Child undernutrition, tropical enteropathy, toilets, and handwashing. Lancet 2009;374(9694):1032-5.

8. Hoddinott J, Alderman H, Behrman JR, Haddad L, Horton S. The economic rationale for investing in stunting reduction. Matern Child Nutr 2013;9:69-82.

9. Hoddinott J, Behrman JR, Maluccio JA, Melgar P, Quisumbing AR, et al. Adult consequences of growth failure in early childhood. Am J Clin Nutr 2013;98(5):1170-8.

10. Grantham-McGregor S, Cheung YB, Cueto S, Glewwe P, Richter L, et al. Developmental potential in the first 5 years for children in developing countries. Lancet 2007;369(9555):60-70.

11. Spears D, Ghosh A, Cumming 0. Open defecation and childhood stunting in India: an ecological analysis of new data from 112 districts. PLoS One 2013;8(9):e73784.

12. Horwood P, Greenhill A. Cholera in Papua New Guinea and the importance of safe water sources and sanitation. Western Pac Surveill Response J 2012;3(1):3-5.

13. World Health Organization. Constitution of the World Health Organization, Geneva, Switzerland, 1948.

14. EFA Global Monitoring Report team. EFA Global Monitoring Report: Education For All 2000-2015: Achievements and Challenges. Paris, France: UNESCO Publishing, 2015.

15. UNICEF. Advancing WASH in schools monitoring. New York, USA: UNICEF, 2015.

16. Jasper C, Le TT, Bartram J. Water and sanitation in schools: A systematic review of the health and educational outcomes. Int J Environ Res Public Health 2012;9(8):2772-87.

17. Sommer M, Kjellén M, Pensulo C. Girls' and women's unmet needs for menstrual hygiene management (MHM): The interactions between MHM and sanitation systems in low-income countries. J WASH Dev 2013;3(3):283-7.

18. Open working group of the general assembly on sustainable development goals. Open working group proposal for sustainable development goals. New York, NY: United Nations, 2015.

19. Jagals P, Nala N, Tsubane T, Moabi M, Motaung K. Measuring changes in water-related health and hygiene practices 
by developing-community households. Water Sci Technol 2004;50(1):91-7.

20. Coffey D, Gupta A, Hathi P, Khurana N, Spears D, et al. Revealed preference for open defecation. Econ Polit Weekly 2014;49(38):43-55.

21. Clasen T, Boisson S, Routray P, Torondel B, Bell M, et al. Effectiveness of a rural sanitation programme on diarrhoea, soil-transmitted helminth infection, and child malnutrition in Odisha, India: A cluster-randomised trial. Lancet Glob Health 2014;2(11):e645-53.
22. Mara D. Sanitation: What's the real problem? IDS Bull-Inst Dev Stud 2012;43(2):86-92.

23. Esrey SA, Potash JB, Roberts L, Shiff C. Effects of improved water-supply and sanitation on ascariasis, diarrhea, dracunculiasis, hookworm infection, schistosomiasis, and trachome. Bull World Health Organ 1991;69(5): 609-21.

24. Department of National Planning and Monitoring. National water, sanitation and hygiene (WaSH) policy 2015-2030. Port Moresby, Papua New Guinea, 2015. 